\title{
Three-Robot Minimax Travel-Distance Optimal Formation
}

\author{
Zhenchao Jia ${ }^{\dagger}$, Hongbin $\mathrm{Ma}^{* \dagger}$, Chenguang Yang ${ }^{\ddagger}$, Meiling Wang ${ }^{\dagger}$
}

\begin{abstract}
Multi-robot formation problem has received increasing attention due to its wide applications such as surveillances and various services. To illustrate a novel framework on optimal multi-robot formation given in our previous work, which aims to answer the long-term ignored fundamental problem of describing the formation and clarifying optimal formation rigorously in a mathematical manner, as a preliminary case study, various cases of the simplest optimal line formation of three robots, i.e. minimax travel-distance line formation problems, where each robot admits to move with the same constant speed along any chosen direction and the three-robot team aims to row on a straight line with the minimum maximal travel distance, are investigated in this note. Such problems look like very easy to resolve, however, to our surprise, mathematical results for these cases established with geometric analysis and inequalities have shown the non-trivialness of the most simple optimal line formation problem. Extensive simulations have also been conducted and briefly reported in this contribution, and these experimental results are found to coincide with the established theoretical results.
\end{abstract}

\section{INTRODUCTION}

In the past decades, multi-robot systems have received increasing attention in the research community due to their wide applications and academic challenges, especially, multirobot systems can be regarded as important background of multi-agent systems [1], which are often used to model the so-called complex systems [2]. For multi-robot systems, or generally multi-agent systems, which consist of many individual agents, one challenging big academic problem, which has been extensively addressed in the literatures from various different aspects, is to investigate the intrinsic relationship between the individuals and the whole, i.e. whether individual behaviors can result in some global properties, such as stability [3], consensus [4], synchronization [5], formation [6], [7], [8], [9], [10], [11], [12], [13], [14], [15], [16], [17], and so on. In many applications of multiple robots such as cleaning, mine sweeping, security patrols, and so on, robot formation plays one important and basic role in sense that the robots in a team are usually controlled as a whole to follow required formations to accomplish tasks with satisfied overall performance.

Many different issues about robot formation and formation control, e.g. safety in formation, stability of the formation, etc., have been extensively studied in the literature, some of which have been briefly surveyed in [18] by Y.Q.Chen and Z.M.Wang.

Despite of existing extensive studies and increasing research interests on formation control, we notice that the following two fundamental problems are seldom exactly addressed and discussed in a mathematical manner: How to define a formation? How to define the 'optimal' formation?

$\dagger$ ZZhenchao Jia, Hongbin Ma and Meiling Wang $\}$ are with School of Automation, Beijing Institution of Technology, Beijing 100081, China.

$\ddagger$ Chenguang Yang is with School of Computing and Mathematics, University of Plymouth, Plymouth PL4 8AA, United Kingdom.

* Corresponding author. Email: mathmhb@bit.edu.cn.

This work is partially supported by National Nature Science Foundation (NSFC) under Grants 61004059, 61004139 and 60904086. Also supported by Program for New Century Excellent Talents in University (NCET-090045,NCET-10-0046), BIT Scientific Base Supporting Funding, and BIT Basic Research Funding.
Motivated by the above questions and many practical demands for efficient robot formation, in our previous work [19], some preliminary basic concepts related to robot formation have been mathematically defined and a new framework of general optimal formation problem has been introduced to lay a solid foundation for future research on multi-robot optimal formation.

In this paper, under the established framework of robot formation, the minimax travel-distance optimal line formation problem is discussed for several typical cases with mathematical results presented for the optimal solutions. As a preliminary study, this paper will focus on several cases of optimal line formation of a three-robot group, where the desired formations are raw line formation, fixed-slope line formation, and ordered line formation, respectively, since line formation is a typical class of frequently demanded formations and can serve as a basis to investigate more general formations of multiple robots.

Roughly speaking, the more the constraint conditions of a desired formation are imposed, the smaller the formation set meeting the requirement is. In almost all interested cases, the formation set usually consist of infinite many possible formations, hence generally it is difficult to figure out the optimal one and require great amount of computation efforts to seek the optimal formation process involving dynamics of all robots. For the optimal line formation problems considered in this contribution, instead of blind search for the optimal solution, geometrical method is skillfully adopted here to analytically solve these problems. Note that although the line formation looks like very simple and easy to solve, our nontrivial analysis to several typical case studies with geometrical arguments as well as trigonometric inequalities has shown that, generally speaking, solving the optimal formation problem is far from obvious and very challenging in terms of technical difficulties involved and computation complexity. follows:

For clarity, contributions of this paper are highlighted as

- The minimax travel-distance raw line formation of three-robot group is analytically discussed, with detailed mathematical proofs.

- Optimal solutions to the minimax travel-distance fixedangle line formation problem and ordered line formation problem of three-robot group are also presented, whose nontrivial proofs are omitted here to save space.

- Simulation results have verified our theoretical results for three-robot line formation and these preliminary case studies have obvious practical background and potential applications, which may serve as a basis for discussing more general optimal formation problems.

The remainder of this paper is organized as follows: first, for convenience of readers, some preliminary mathematical concepts related with robot formation are given in Section II as well as our general framework of optimal multi-robot formation; then, mathematical results on optimal three-robot line formation problems for three typical cases are rigorously presented in Section III, as well as some simulation results given in Section IV which verify our theoretical results; 
finally, we conclude this paper in Section $\mathrm{V}$ with several concluding remarks.

\section{Preliminaries: CONCEPTS AND FramewORK}

For brevity and convenience, we sketch some preliminary concepts and the framework of optimal multi-robot formation, which is detailedly discussed in our previous work [19].

\section{A. Preliminary Concepts}

Definition 2.1: Suppose that the robot moves in one subset $\Omega$ of the whole euclidean space $\mathcal{R}^{m}$, where $m$ is usually taken as 2 or 3 in practical life. Here $\Omega \subseteq \mathcal{R}^{m}$ is called free space of the robot. If $\Omega=\mathcal{R}^{m}$, then we say that the robot is moving unconstrainedly.

Definition 2.2: Any mapping $f: \mathcal{S} \rightarrow \mathcal{R}^{1}$ is called a scalar function of group status. Any mapping $f: \mathcal{S} \rightarrow \mathcal{R}^{d}$ is called a vector function of group status.

Given a function $f$ of group status, and suppose that robot $R_{i}$ move to $P_{i}(t)$ at time $t(i=1,2, \cdots, n)$, then $f(S(t))$ is in fact a value depending on all robots' positions. For convenience, we use $d(P, Q)$ to denote the distance between points $P$ and $Q$ in euclidean space $\mathcal{R}^{m}$. That is to say, in case of $m=2$,

$$
d(P, Q) \triangleq \sqrt{\left(x_{P}-x_{Q}\right)^{2}+\left(y_{P}-y_{Q}\right)^{2}}
$$

where $\left(x_{P}, y_{P}\right)$ and $\left(x_{Q}, y_{Q}\right)$ are coordinates of points $P$ and $Q$, respectively. In case of $m=2$, we also use $\alpha(P, Q)$ to denote the slope angle of vector $\overrightarrow{P Q}$, which can be defined by

$$
\alpha(P, Q) \triangleq \arctan \left(x_{Q}-x_{P}, y_{Q}-y_{P}\right)
$$

where $\arctan (x, y) \in \mathcal{R}[2 \pi]$ denotes the arctangent angle in the correct quadrant determined by the coordination $(x, y)$. Function $d_{i j}(\cdot): \mathcal{S} \rightarrow \mathcal{R}^{1}$ is defined by

$$
d_{i j}(S(t)) \triangleq d\left(P_{i}(t), P_{j}(t)\right)
$$

Besides, we need the follow notations:

$$
\begin{array}{rll}
\alpha_{i}(S(t) ; Q) & \triangleq & \triangleq \\
\alpha_{i j}(S(t)) & \triangleq & \alpha\left(P_{i}(t), P_{j}(t)\right) \\
\beta_{i j}(S(t) ; Q) & \triangleq & \alpha\left(Q, P_{j}(t)\right)-\alpha\left(Q, P_{i}(t)\right) \\
\beta_{i k j}(S(t)) & \triangleq & \alpha\left(P_{k}(t), P_{j}(t)\right)-\alpha\left(P_{k}(t), P_{i}(t)\right)
\end{array}
$$

Definition 2.3: For a group of robots $R_{1}, R_{2}, \cdots, R_{n}$, suppose that the group status $S(t) \in \Omega^{n}$ is determined by a time-dependent mapping $G_{t}: \mathcal{S} \times \Theta$ :

$$
S(t)=G_{t}(S(0), \theta), \theta \in \Theta_{\mathcal{G}}
$$

where $S(0)$ is the initial group status of robots, and $\theta \in \Theta_{\mathcal{G}}$ is a vector holding parameters of the robots motion. Then we say that $G_{t}$ is a group motion model of the robots and $\theta$ is called the motion parameter vector of the robots.

Definition 2.4: For a group of robots $R_{1}, R_{2}, \cdots, R_{n}$, suppose that all robots share the same motion model, that is to say, each robot's position $P_{i}(t) \in \Omega$ at time $t$ is determined by a time-dependent mapping $F_{t}: \Omega \times \Theta$ :

$$
P_{i}(t)=F_{t}\left(P_{i}(0), \theta_{i}\right), \theta_{i} \in \Theta
$$

where $\Theta$ is a common set of parameter vectors for all robots and $P_{i}(0)$ is the initial position of Robot $R_{i}$. In this case, $F_{t}$ is a homogeneous motion model of the robots and $\theta_{i} \in \Theta$ is the motion parameter of Robot $R_{i}$.

Definition 2.5: For a group of robots $R_{1}, R_{2}, \cdots, R_{n}$, suppose that all robots share the same motion model and each robot will not move any longer once stop at certain time instant, that is to say, each robot's position $P_{i}(t) \in \Omega$ at time $t$ is determined by a mapping $F: \mathcal{R} \times \Omega \times \Theta$ such that

$$
P_{i}(t)= \begin{cases}F\left(t ; P_{i}(0), \theta_{i}\right), & \text { if } \quad 0 \leq t \leq T_{i} \\ F\left(T_{i} ; P_{i}(0), \theta_{i}\right), & \text { if } \quad t \geq T_{i}\end{cases}
$$

where $\Theta$ is a common set of parameter vectors for all robots and $P_{i}(0)$ is the initial position of Robot $R_{i}$. In this case, $F$ is called a truncatable homogeneous motion model of the robots and $\theta_{i} \in \Theta$ is the motion parameter of Robot $R_{i}$. Note that the truncation time (or termination time) $T_{i}$ generally may be dependent of $\theta_{i}$.

\section{B. What is a formation?}

Definition 2.6: For a group of robots $R_{1}, R_{2}, \cdots, R_{n}$, a prescribed formation set $\mathcal{F}$ is essentially a set of group status which satisfy certain specific constraints. Mathematically speaking, suppose that

$$
g_{1}, g_{2}, \cdots, g_{p}, h_{1}, h_{2}, \cdots, h_{q}: \mathcal{S} \rightarrow \mathcal{R}
$$

are several given functions of group status, then the set

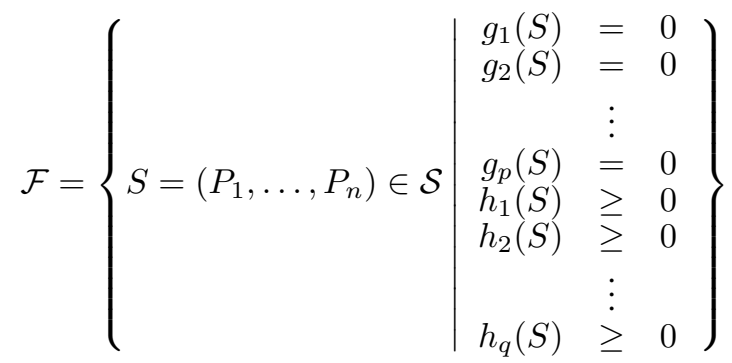

is called a formation set, which is defined by equality constraints $g_{j}(S)=0(j=1,2, \cdots, p)$ and inequality constraints $h_{j}(S) \geq 0(j=1,2, \cdots, q)$. And any element of $\mathcal{F}$ is called a desired formation.

\section{What is optimal formation?}

Definition 2.7: Mathematically speaking, a formation process $\mathcal{P}$ is an indexed collection $\{S(t), t \geq 0\}$ of group status $S(t)$, ending with or approaching to a desired formation. Intuitively speaking, a formation process is the whole process of a group of robots starting from their initial group status until they formulate a desired formation.

Definition 2.8: Given a formation set $\mathcal{F}$ and a formation process $\mathcal{P}=\{S(t), t \geq 0\}$, let

$$
d_{\text {close }}(t) \triangleq \inf _{S_{F} \in \mathcal{F}} d\left(S(t), S_{F}\right)
$$

where $d\left(S(t), S_{F}\right)$ denotes the distance between group status $S(t)$ and $S_{F}$, which will be discussed later and which intuitively measures the degree of closeness to the desired formation.

Definition 2.9: Let $S=\left(P_{1}, P_{2}, \cdots, P_{n}\right) \in \Omega^{n}$ and $S^{\prime}=\left(P_{1}^{\prime}, P_{2}^{\prime}, \cdots, P_{n}^{\prime}\right) \in \Omega^{n}$ be two group status. Then the distance between $S$ and $S^{\prime}$ can be defined as

$$
d\left(S, S^{\prime}\right) \triangleq \max \left(d\left(P_{1}, P_{1}^{\prime}\right), d\left(P_{2}, P_{2}^{\prime}\right), \cdots, d\left(P_{n}, P_{n}^{\prime}\right)\right)
$$


where $d\left(P_{i}, P_{i}^{\prime}\right)$ denotes the euclidean distance between point $P_{i}$ and $P_{i}^{\prime}$ in $\mathcal{R}^{m}$. It is easy to verify that

$$
d(S, S)=0 ; d\left(S, S^{\prime}\right)=d\left(S^{\prime}, S\right) ; d\left(S, S^{\prime}\right)=0 \text { iff } S=S^{\prime}
$$

and the triangular inequality

$$
d\left(S, S^{\prime}\right)+d\left(S^{\prime}, S^{\prime \prime}\right) \geq d\left(S, S^{\prime \prime}\right)
$$

holds for any group status $S, S^{\prime}, S^{\prime \prime} \in \Omega^{n}$. Hence $d(\cdot, \cdot)$ defines a distance well in $\Omega^{n}$.

Definition 2.10: Any scalar function $I(\mathcal{P})$ of a formation process $\mathcal{P}$ can be regarded as a performance index of the formation process, which presents a criterion for quantitatively evaluating the formation process.

Example 2.1: For a finite-time formation process $\mathcal{P}$, let $D_{i}$ denotes the total travel distance of Robot $R_{i}$ from initial time to termination time $T_{i}$. Maximum travel distance $D$ is defined as

$$
D \triangleq \max \left(D_{1}, D_{2}, \cdots, D_{n}\right)
$$

Obviously, $D$ is a scalar determined by the formation process, hence, maximum travel distance $D(\mathcal{P})$ can be regarded as a performance index of the formation process $\mathcal{P}$.

Definition 2.11: Given a formation set $\mathcal{F}$, a performance index $I(\cdot)$, a group motion model $G_{t}$, and a set $\mathbb{P}$ of formation processes (following the specified motion model) starting from initial group status $S_{0}$, then the purpose of general optimal formation problem is to find an optimal formation process $\mathcal{P}_{\text {opt }}$ which minimizes the performance index $I(\mathcal{P})$, i.e.

$$
\mathcal{P}_{\text {opt }}=\underset{\mathcal{P} \in \mathbb{P}}{\arg \min } I(\mathcal{P})
$$

And

$$
I^{*} \triangleq I\left(\mathcal{P}_{\text {opt }}\right)
$$

is called the optimal performance index of this optimal formation problem.

When we consider parametric motion models and formation processes, the optimal formation process is indeed to find the optimal formation parameters $\theta^{*} \in \Theta$ such that

$$
\theta^{*}=\underset{\theta \in \Theta}{\arg \min } I\left(S_{0} ; \Theta\right)
$$

where

$$
I\left(S_{0} ; \theta\right) \triangleq I\left(\mathcal{P}\left(S_{0} ; \theta\right)\right)
$$

is essentially determined by the initial group status as well as the motion parameters $\theta \in \Theta$, and consequently the optimal performance index is

$$
I^{*}=I\left(S_{0} ; \theta\right)
$$

Definition 2.12: In Definition 2.11, if the performance index is taken as the maximum travel distance $D(\mathcal{P})$ given in Example 2.1, then the optimal formation problem is called minimax travel-distance optimal formation problem, or simply minimax travel-distance formation problem.

The formulation of optimal formation problems looks like very simple from Definition 2.11, however, from this contribution, we can see that such a class of formation problems are generally very nontrivial even for the most simple problems of three-robot optimal line formations. Based on the concepts given above, we are ready to present the minimax travel-distance three-robot line formation problem.

\section{Minimax Travel-Distance Three-Robot Line FORMATION}

\section{A. Considered Line Formations}

In this paper, we focus on the following three typical line formations.

Definition 3.1: (Raw) Line Formation: A (raw) line formation refers to the group status such that all robots are exactly located on the same straight line. Such a formation set can be mathematically defined by

$$
\mathcal{F}=\left\{S \mid \beta_{i j k}(S)=0, \forall 1 \leq i<j<k \leq n\right\}
$$

or, explicitly, the constraints in the plane can be reduced to $\left(x_{1}-x_{2}\right)\left(y_{1}-y_{i}\right)-\left(x_{1}-x_{i}\right)\left(y_{1}-y_{2}\right)=0, i=3,4, \ldots, n$ where $\left(x_{i}, y_{i}\right)$ denotes the desired position of Robot $R_{i}$.

In many situations, the desired line formation needs more additional constraints, some of which have been described in [19], here we consider only fixed-slope line formation and ordered line formation.

Definition 3.2: Fixed-Slope Line Formation: Suppose that all robots are expected to follow a line with certain slope $\kappa_{\mathcal{F}}=\tan \gamma_{\mathcal{F}}$, where $\gamma_{\mathcal{F}}$ is the desired slope angle. Then, the formation set can be mathematically defined by

$$
\mathcal{F}=\left\{S \in \mathcal{S} \mid \alpha_{i j}(S)=\kappa_{\mathcal{F}}, \forall 1 \leq i<j<k \leq n\right\}
$$

Definition 3.3: Ordered Line Formation: Suppose that all robots are expected to follow a line with specified alignment order

$$
R_{1} \rightarrow R_{2} \rightarrow \ldots \rightarrow R_{n}
$$

Then, the formation set can be mathematically defined by

$$
\begin{gathered}
\mathcal{F}=\left\{S \in \mathcal{S} \mid \quad \beta_{j i k}(S)=0, d_{i k}(S)-d_{i j}(S) \geq 0,\right. \\
\forall 1 \leq i<j<k \leq n\}
\end{gathered}
$$

As a starting point, we only consider optimal line formation of a three-robot group, which is the most simple case and may play a basic role for understanding optimal formation of more robots since a group of many robots may be divided in sub-groups with no more than three robots, which may help to seek global optimal formation by divideand-conquer strategy.

\section{B. Notations For Analysis}

As to the performance index, we consider the maximal travel-distance $\mathcal{D}(\mathcal{P})$, which intuitively reflects maximal cost for robots to reach a desired formation. For robots moving with the same speed, this performance index will be proportional to the formation time, hence solving minimax traveldistance line formation problem is equivalent to solving minimum time line formation problem. For the three typical desired line formations given in Definitions 3.1-3.3, we are ready to investigate the corresponding minimax traveldistance three-robot line formation with any initial group status $S(0)$, and we will present its optimal solution by stating explicitly the desired target position of each robot and the optimal performance index, i.e., maximal travel distance, will be analytically figured out. For the line formation problems considered here, the optimal formation process can be generated as follows:

1) Compute every robot's optimal target position in the optimal formation, then we can get the set $\mathcal{F}^{*}$ of desired optimal formations and the corresponding optimal formation parameters $\theta^{*}$.

2) Robots go straight to their optimal target position in a constant speed, and stop after reaching their optimal target position. 
3) Once the last robot reaches its optimal target position, the minimax travel-distance formation has formed.

To facilitate our theoretical analysis, we need to introduce some notations first, which are given below as illustrated in Fig.1:

1) Three robots are denoted by $R_{1}, R_{2}$, and $R_{3}$, respectively. Initially, three robots usually constitute a triangle, whose vertices are also named as $R_{i}(i=$ $1,2,3)$ without confusion.

2) Let $f \in \mathcal{F}$ denote any straight line, i.e. one possible desired formation, then the so-called optimal line formation problem is in fact to determine an optimal line $f^{*}$ such that three robots can locate on the line $f^{*}$ by moving for some time.

3) For simplicity and convenience, let

$$
d_{i}(f) \triangleq d_{i}(S(0), f)
$$

be the initial distance between $R_{i}$ and $f$ for any given desired straight line $f$.

4) Let

$$
l_{1}=d_{23}(S(0)), l_{2}=d_{13}(S(0)), l_{3}=d_{12}(S(0))
$$

be each two robots' initial distance.

5) Let

$$
\begin{aligned}
& h_{1}=d_{1}\left(S(0), \overline{R_{2} R_{3}}\right) \\
& h_{2}=d_{2}\left(S(0), \overline{R_{1} R_{3}}\right) \\
& h_{3}=d_{3}\left(S(0), \overline{R_{1} R_{2}}\right)
\end{aligned}
$$

where

$$
\overline{R_{2} R_{3}}, \overline{R_{1} R_{3}}, \overline{R_{1} R_{2}}
$$

are the lines on which each two robots are located.

With the notations above, given a line $f$, there will be a set $\left\{d_{1}(f), d_{2}(f), d_{3}(f)\right\}$. Let

$$
D_{\max }(f)=\max \left\{d_{1}(f), d_{2}(f), d_{3}(f)\right\}
$$

Therefore, to obtain the minimax travel-distance $D^{*}$, we should find a line $f^{*}$ such that

$$
D^{*}=\min _{f \in \mathcal{F}} D_{\max }(f)=D_{\max }\left(f^{*}\right)
$$

which consequently determines the optimal formation parameters $\theta^{*}$ for three robots, in the way that the robots should go to the desired line with the shortest path, as indicated by $d_{i}(f), i=1,2,3$. Next, we give our conclusions of the optimal problems.

\section{C. (Raw) Line Formation}

Theorem 3.1: For a three-robot group with any initial group status $S(0)$, if their desired formation is raw line formation given in Definition 3.1, then

(i) The minimax travel-distance $D^{*}$ can be explicitly given by

$$
D^{*}=\frac{1}{2} h_{\min }
$$

where $h_{\min }$ is defined as

$$
h_{\text {min }} \triangleq \min \left\{h_{1}, h_{2}, h_{3}\right\}
$$

(ii) The corresponding optimal formation $f^{*}$ is the perpendicular bisector of the shortest altitude of the triangle $R_{1} R_{2} R_{3}$.

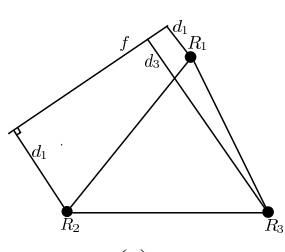

(a)

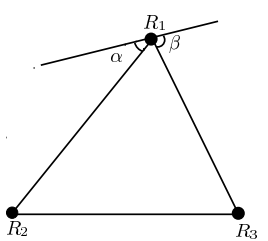

(b)

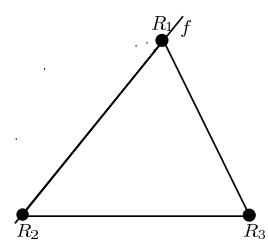

(c)

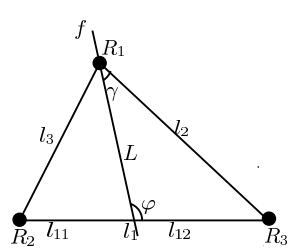

(d)

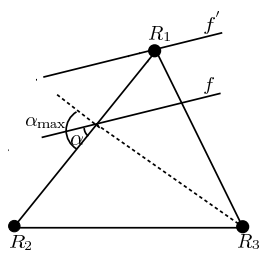

(e)
Fig. 1. The situation of the relationship of the triangle's vertex and the line $f$

Remark 3.1: Raw line formation is the most basic line formation, which contains least constraints in the formation set $\mathcal{F}$, hence the corresponding formation set $\mathcal{F}$ is much larger than other line formations.

Proof. According to the relative position relation between the line $f$ and the triangle, we can divide all possible situations into five cases:

1) The line $f$ does not intersect with the triangle, as shown in Fig.1(a);

2) The line $f$ passes only a vertex of the triangle, as shown in Fig.1(b);

3) An side of the triangle is on the line $f$, as shown in Fig.1(c);

4) The line $f$ passes a vertex of the triangle and intersects with an side of the triangle, as shown in Fig.1(d);

5) The line $f$ intersects with two sides of the triangle, as shown in Fig.1(e).

To establish Theorem 3.1, we need only to discuss five cases shown in Fig. 1(a)-Fig. 1(e), respectively, and show that the lower bound $\frac{h_{\min }}{2}$ of $D_{\max }$ can be achieved in certain case, while in other cases we must have $D_{\max }>\frac{h_{\min }}{2}$. The key idea is to adopt the following basic relationship among the heights $h_{i}$, side lengths $l_{i}$ and angles $\angle R_{i}(i=1,2,3)$ :

$$
\begin{gathered}
\left\{\begin{array}{l}
h_{1}=l_{3} \sin \angle R_{2}=l_{2} \sin \angle R_{3} \\
h_{2}=l_{3} \sin \angle R_{1} \\
h_{3}=l_{2} \sin \alpha
\end{array}\right. \\
\left\{\begin{array}{l}
d_{2}=l_{3} \sin \alpha \\
d_{3}=l_{2} \sin \left(\alpha+\angle R_{1}\right)=l_{2} \sin \beta
\end{array}\right.
\end{gathered}
$$

where $\beta=\pi-\alpha-\angle R_{1}$. Since the proof details are rather involved, we omit them to save space due to the page limitation.

\section{Fixed-Slope Line Formation}

Theorem 3.2: For a three-robot group with any initial group status $S(0)$, if their desired formation is fixed-slope line formation given in Definition 3.2, suppose that the desired slope angle is $\gamma$ w.r.t. the line $R_{2} R_{3}$, as shown in Fig. 2, then

(i) The minimax travel-distance $D^{*}$ is

$$
D^{*}=\frac{1}{2} \max \left\{l_{3} \sin \left(\angle R_{2}-\gamma\right), l_{2} \sin \left(\angle R_{3}+\gamma\right)\right\} \text {. }
$$


(ii) The corresponding optimal formation $f^{*}$ is the line with specified slope angle passing the midpoint of the side $\overline{R_{1} R_{2}}$ if

$$
\begin{aligned}
& l_{3} \sin \left(\angle R_{2}-\gamma\right) \geq l_{2} \sin \left(\angle R_{3}+\gamma\right), \\
& \text { or } \overline{R_{1} R_{3}} \text { if } \\
& l_{3} \sin \left(\angle R_{2}-\gamma\right) \leq l_{2} \sin \left(\angle R_{3}+\gamma\right) .
\end{aligned}
$$

There are two optimal formations when

$$
l_{3} \sin \left(\angle R_{2}-\gamma\right)=l_{2} \sin \left(\angle R_{3}+\gamma\right) .
$$

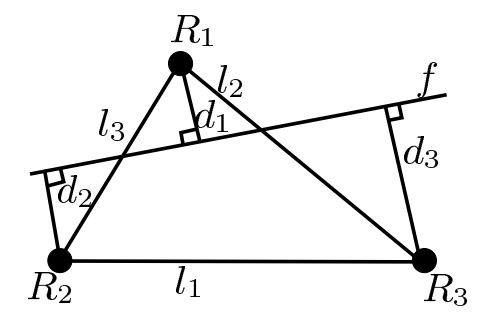

Fig. 2. The relationship of $l_{i}, d_{i}$, and $\angle R_{i}(i=1,2,3)$ in optimal fixedslope line formation problem

Remark 3.2: We can show that the optimal value $D^{*}$ in this theorem will not be smaller than $\frac{1}{2} h_{\min }$, which is the optimal value obtained in Theorem 3.1. This non-obvious fact is intuitive since fixed-slope line formation adds some constraints to the raw line formation.

\section{E. Ordered Line Formation}

In this formation, three robots aim to form a straight line with a fixed order, say

$$
R_{1} \rightarrow R_{2} \rightarrow R_{3}
$$

without loss of generality. That is to say, robot $R_{2}$ should be in the middle of the other two robots. In this paper, we suppose that any two robots can gather at the same place.

Theorem 3.3: For a three-robot group with any initial group status $S(0)$, if their desired formation is ordered line formation given in Definition 3.3, then

(i) The minimax travel-distance $D^{*}$ is

$$
D^{*}= \begin{cases}\frac{h_{2}}{2} & \text { if } \max \left\{\angle R_{1}, \angle R_{3}\right\}<\frac{\pi}{2} \\ \frac{1}{2} \min \left\{\overline{R_{1} R_{2}}, \overline{R_{2} R_{3}}\right\} & \text { if } \max \left\{\angle R_{1}, \angle R_{3}\right\} \geq \frac{\pi}{2}\end{cases}
$$

(ii) If

$$
\max \left\{\angle R_{1}, \angle R_{3}\right\}<\frac{\pi}{2}
$$

then the corresponding optimal formation $f^{*}$ is the perpendicular bisector of the altitude of the side $\overline{R_{1} R_{3}}$; otherwise, the corresponding optimal formation $f^{*}$ is the shorter one side among sides $\overline{R_{1} R_{2}}$ and $\overline{R_{2} R_{3}}$.

\section{Simulation Results}

In last section, we have presented three conclusions about minimax travel-distance three-robot line formation problems. We will make some simulation experiments to clearly verify these conclusions in this section.

In the plane, the initial positions of three robots can be arbitrarily set to make them form different types of triangles. After setting the initial groups status, we may use the theoretical results given in last section to calculate optimal $D^{*}$ and the corresponding optimal desired line formation $f^{*}$. To verify the validness of the theoretical results, for other

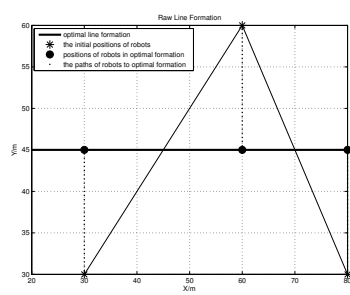

(a)

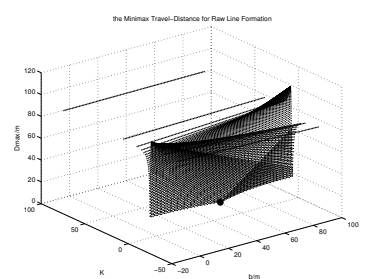

(b)
Fig. 3. The case of line formation problem

arbitrarily chosen line $f$, whose equation can be expressed in the two-dimensional Cartesian coordinate system with two parameters $k$ and $b$ as follows

$$
y=k x+b,
$$

we can calculate the maximal distance $D_{\max }(f)=$ $\max \left\{d_{1}(f), d_{2}(f), d_{3}(f)\right\}$, and then check whether $D_{\max }(f) \geq D^{*}$. By changing the parameters $k$ and $b$ in a large range, we need only verify that $D_{\max }(f) \geq D^{*}$ always hold.

a) Raw Line Formation: The three robots' initial positions are $R_{1}(30,30), R_{2}(60,60)$, and $R_{3}(80,30)$, respectively. Then, we can calculate the length of three sides as follows

$$
\begin{aligned}
& l_{1}=10 \sqrt{13} \\
& l_{2}=50 \\
& l_{3}=30 \sqrt{2}
\end{aligned}
$$

Obviously, $l_{2}$ is the longest side. Then from Theorem 3.1, we know that the line $f^{*}$ is the triangle's median line and passes the point $(45,45)$ and the point $(70,45)$. Consequently, we obtain that the line $f^{*}$ has parameters $K=0$ and $b=45$, i.e. the line $f^{*}$ can be described by

$$
y=45
$$

Then we know that distance $D^{*}$ is 15 . Finally we get the result shown in Fig.3(a), where the thick solid line is line $f^{*}$, and the big points denote the robots' positions in the optimal formation, and the dotted lines denote the robots' moving paths from their initial position to the optimal formation.

Next, we will calculate maximal distance $D_{\max }$ between three robots and an arbitrary line $f$. The slope $K$ of the line $f$ varies from -40 to 100 by a step of 1.4 , and for each certain $K$, we change $b$ from -20 to 100 by a step of 1.2 . For every line $f$ corresponding to each parameter pair $(K, b)$, we calculate the distances $d_{1}, d_{2}, d_{3}$ and $D_{\max }$, whose values are plotted vs. $K$ and $b$ in Fig.3(b). As shown in Fig.3(b), X axis denotes the value $b, \mathrm{Y}$ axis denotes the value $k$, and $\mathrm{Z}$ axis denotes the value $D_{\max }$. As is seen, the big black point is $D^{*}$, and it is the same as our theoretical result, which verifies the validity of the theoretical analysis.

b) Fixed-Slope Line Formation: The results of the fixed-slope line formation problem of three-robot group are shown in Fig.4(a) and Fig.4(b). The three robots' initial positions are still $(30,30),(60,60)$, and $(80,30)$. And we fix the slope of desired formation as

$$
k=0.5
$$

In Fig.4(a), it is shown that the line $f^{*}$ is

$$
y=0.5 x+10
$$




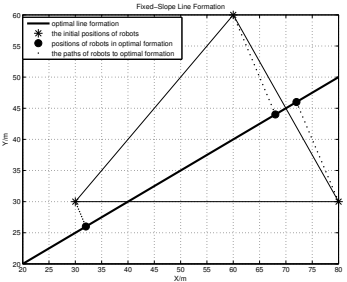

(a)

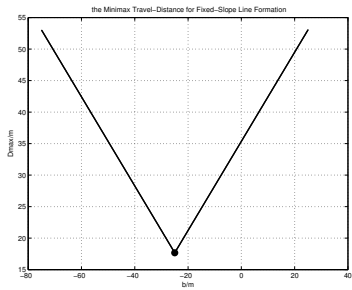

(b)
Fig. 4. The case of fixed-slope line formation problem

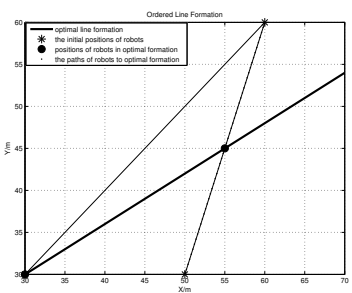

(a)

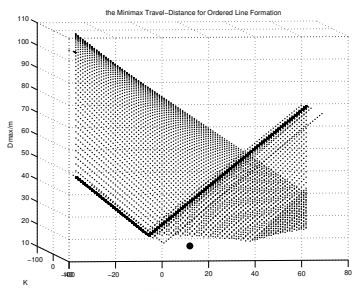

(b)
Fig. 5. The case of ordered line formation problem

which is obtained from Theorem 3.2.

Fig.4(b) depicts the distance $D_{\max }$ between three robots and an arbitrary line $f$, and we use similar method to change the line $f$ by changing the parameter $b$. As is shown in Fig.4(b), $\mathrm{X}$ axis denotes the value $b$, and $\mathrm{Y}$ axis denotes the value $D_{\max }$. The optimal formation is the same with the theoretical result given by Theorem 3.2, which shows consistence between the theory and the experiments.

c) Ordered Line Formation: The results of ordered line formation problem of three-robot group are shown in Fig.5(a) and Fig.5(b). We just do experiments in the case of the angle $\angle R_{2}<\frac{\pi}{2}$, because it has the same conclusion with the raw line formation problem when the angle $\angle R_{2} \geq \frac{\pi}{2}$. Firstly, we set the three robots' initial positions as $R_{1}(30,30), R_{2}(60,60), R_{3}(50,30)$. And we suppose that the desired robot order is $R_{1} \rightarrow R_{2} \rightarrow R_{3}$. That is to say, $R_{2}$ must be in the middle of the $R_{2}$ and $R_{3}$. Next, we obtain the optimal formation $f^{*}$ from Theorem 3.3, which is shown in Fig.5(a). As we can see, $f^{*}$ is the line given by

$$
y=0.6 x+12
$$

Fig.5(b) depicts the distance $D_{\max }$ for each $f$ which is changed in a similar way with the method above. And it verifies the validness of the theoretical analysis.

\section{Conclusion}

To illustrate the mathematical framework of optimal multi-robot formation introduced in our previous work [19], this paper focuses on the most simple cases of three-robot line formations where the robots moving with the same speed are expected to row on one straight line with the minimum maximal travel-distance so that the formation can be formulated in the most efficient way. Non-trivial mathematical results to the optimal solutions for these problems have been presented, which illustrate some challenges in solving such optimal formation problems. The theoretical results are also verified through extensive simulation results, which validify correctness of the established mathematical results and indicate alternative approaches to find the solutions of more general optimal formation problems due to the mathematical difficulties involved.
As a starting point in this direction, more work need to be done in the future to address the more challenging problems for more robots, more formation shapes, more formation cases, more formation indices, more motion models, and so on. And even for three-robot minimum-time formation problems, there are some remaining challenging problems, for example, the geometric methods in this contribution may not be suitable to the cases where the robots have different speeds. And currently we do not consider more challenging optimal/sub-optimal decentralized formation algorithms, which need more investigations in the future.

\section{REFERENCES}

[1] R. Olfati-Saber, J. A. Fax, and R. M. Murray. Consensus and cooperation in networked multi-agent systems. Proceedings of the IEEE, 95(1):215-233, Jan. 2007.

[2] J. H. Holland. Hidden Order: How Adaptation Builds Complexity. Addison-Wesley, New York, 1996.

[3] H. B. Ma, K. Y. Lum, and S. S. Ge. Decentralized Aström-Wittenmark self-tuning regulator of a multi-agent uncertain coupled ARMAX system. In Proceedings of the 2007 IEEE Multi-conference on Systems and Control, pages 363-368, Suntec City Convention Centre, Singapore, October 1-3 2007.

[4] R. Olfati-Saber and R. M. Murray. Consensus problems in networks of agents with switching topology and time-delays. IEEE Transactions on Automatic Control, 49:1520-1533, September 2004.

[5] H. B. Ma. Decentralized adaptive synchronization of a stochastic discrete-time multi-agent dynamic model. SIAM Journal of Control and Optimization, 48(2):859-880, 2009. Published February 25, 2009.

[6] J. P. Desai and J. Ostrowski. Controlling formations of multiple mobile robots. In IEEE International Conference on Robotics and Automation, pages 157-162, 2001.

[7] R. Olfati-Saber and R. M. Murray. Graph rigidity and distributed formation stabilization of multi-vehicle systems. In IEEE International Conference on Decision and Control, pages 2965-2971, 2002.

[8] B. D. O. Anderson and S. Dasgupta. Control of directed formations with a leader-first follower structure. In Proceedings of IEEE Conference on Decision and Control New Orleans, pages 12-14, 2007.

[9] S. Sandeep and B. Fidan. Decentralized cohesive motion control of multi-agent formations. In Proceedings of Mediterranean Conference on Control and Automation, pages 1-6, 2006.

[10] V. T. Ngo and A. Nguyen. Integration of planning and control in robotic formation. In Proceedings of the Australasian Conference on Robotics and Automation, pages 1-8, 2005.

[11] X. P. Yun and G. Alptekin. Formation of distributed physical mobile robots. Journal of Robotic Systems, 14(2):63-67, 1997.

[12] G. Lafferriere, A. Williams, J. Caughman, and J. J. P. Veerman. Decentralized control of vehicle formations. Systems and Control Letters, 54:899-910, 2005.

[13] Y. Hao and S. K. Agrawal. Formation planning and control of ugvs with trailer. Autonomous Robots, 19:257-270, 2005.

[14] A. Serrani. Robust coordinated control of satellite formations subject to gravity perturbations. In Proceedings of the American Control Conference, volume 1, pages 302-307, June 2003.

[15] J. G. Bender. An overview of systems studies of automated highway systems. IEEE Transactions on Vehicular Technology, 40(1):82-99, Feb 1991.

[16] H. Choset. Coverage for robotics: a survey of recent results. Annals of Mathematics and Artificial Intelligence, 31:113-126, 2001.

[17] A. Jadbabaie, J. Lin, and A. S. Morse. Coordination of groups of mobile autonomous agents using nearest neighbor rules. IEEE Transactions on Automatic Control, 48(6):988-1001, June 2003.

[18] Y. Q. Chen and Z. M. Wang. Formation control: A review and a new consideration. In Proceedings of 2005 IEEE/RSJ International Conference on Intelligent Robots and Systems, pages 3181-3186, 2005.

[19] H. B. Ma, M. L. Wang, Z. C. Jia, and C. G. Yang. A new framework of optimal multi-robot formation problem. In Proceedings of 2011 Chinese Control Confernence (CCC2011), Yantai, Jul. 2011. 